\title{
Informal Logic, Argumentation Theory \& Artificial Intelligence
}

\section{Introduction}

\section{Michael A. Gilbert Guest Editor}

York University

As computer technology advances, so does the desire to have computers interact in deeper and more meaningful ways with users. By now everyone has at least filled out a form, booked a flight or hotel, started up a web account, or otherwise communicated with a computer. Most of these interactions are quite straightforward, but not infrequently there is, in some basic, perhaps almost trivial way, a degree of disagreement. When you sign up for a web account and neglect to fill out one required box or request a username or password that does not meet the essential criteria, you are told to go back and do it again. Your application is not accepted, and you are asked to change the offending response. Similarly, when you specify the flights you want for your trip and the results are not to your liking, you go back and change something to get different results. In some sense the activity is one of locating disagreement and working it out. In other words, one might be said to be arguing with the computer, though the quality of dispute leaves much to be desired.

When we think about moving forward with computer-human interaction, it becomes clear that methods for disagreement, for discussion, for locating and resolving differences are essential ingredients for the sophisticated use of machines as artificially intelligent actors. A computer designed to advise on legal issues, consult on health matters, interior decorating, major purchases, travel plans, or what have you, needs to be able to disagree. The computer needs to be able to say, "I don't recommend that, for these reasons," or, "I think your requests/ demands are inconsistent"; in this way the interaction is moved along. Similarly, the human agent needs to be able to disagree with the computer, to request alternatives, to indicate that a position is not understood, and so on. The need for such further activities is well known within the world of theoreticians working on Artificial Intelligence, and especially those in the areas of Natural Language Process- 
ing [NLP], Multi-Agent Systems, and the production of frameworks for rational agents. The first area, as the name indicates, focuses on a computer's ability to use and understand ordinary human language, and within NLP the sub area of argumentation is an essential and crucial component. The second area, MultiAgent Systems, concerns the ability of machines to interact with each other, especially in the solution of problems. The last area, frameworks for rational agents, involves creating models or frameworks in which systems can model human reasoning.

The readers of this journal are well familiar with the complexities of argument, and of the difficulties of teaching its structure and nuances to young students. Imagine, if you will, trying to impart those same skills and techniques to a computer with its literal mode of comprehension and representation. First, an argument must be identified. The very fact of recognizing that there is an argument present requires no small talent, and the second step, identifying what the argument is and putting it into some sort of standard form, requires more. Can a machine identify argument forms? Can it understand argument structure and differentiate between premisses, conclusions, irrelevancies, repetitions, and term variations? Can a machine reliably identify enthymematic components and produce them when necessary? All of this is the essential minimum required for identifying an argument.

As if the complexity of argument identification were not enough, much, much more is required for carrying on an argument. Then, not only is the identification infinitely complicated by the vagaries of human word choice, emphasis, emotion, elliptical and ironic speech, but strategic elements enter as well. In other words, once we are not in a formal or quasi-formal arena, the computer must not only be a competent informal logician, but a rhetorician as well. This means being aware of subtleties in interactions, forming responses to best achieve desired results, and goal orientated argumentation. No easy tasks for anyone, let alone a computer. The systematization of rhetoric is a major step that is necessary for the furtherance of Artificial Intelligence as an interactive undertaking. Thus, the first piece in the issue is Towards Computational Rhetoric by Floriana Grasso.

Grasso's work on rhetorical argument builds upon Perelman and ObrechtsTyteca's concept of audience in the New Rhetoric (1969) to form an "all encompassing framework for the formalization of rhetorical argumentation." This paper is the most technical in the issue, and it is hoped that the reader will persevere, even if daunted by the formulae, in order to appreciate the extent to which a classic work of Argumentation Theory can aid in the furtherance of Artificial Intelligence. Grasso's paper is also presented first as it begins with some helpful background on the area of Natural Language Processing.

Continuing with the theme of Perelman and Obrechts-Tyteca's concept of audience, we turn next to Trevor Bench-Capon who, in his article "Agreeing to Differ: Modelling Persuasive Dialogue Between Parties With Different Values," 
relies on the same concept to discuss argumentation between audiences with differing values. In ethics and law, he explains, it is rare that there are truly compelling arguments, and most often the balance of the argument depends on an audience's consideration of the contrasting values put forward by each side. BenchCapon introduces conceptual matters that allow disputants to accept arguments even though they may disagree as to the inherent values.

It is interesting that two of the essays in this collection draw upon a major philosophical personage in Argumentation Theory. The continual move from the computationally idealized model to something that is practical and can be implemented, requires input from contemporary Argumentation Theory and its practical, grounded approach. Like much of philosophy today, the relationship between the philosophical enterprise and what is being carried out in sibling disciplines is central to both areas.

David Hitchcock's essay, "Pollock On Practical Reasoning," begins from the work of a philosopher who has been deeply involved in computational studies. Hitchcock examines the work of John Pollock's computational architecture for a rational agent, dubbed OSCAR, which is designed to model both practical and theoretical reasoning. Hitchcock argues that the conception of practical reasoning implemented in this architecture is superior to several competing conceptions, but nonetheless suffers from some minor setbacks: "it is solipsistic, it is egoistic and it is unsocial."

Peter McBurney and Simon Parsons' contribution also follows the path of a mix of philosophy, especially in the form of formal dialogue games, and machinemachine interactions. These dialogue games, with their ancient history in philosophy, have, they argue, an important role to play in the understanding and development of multi-agent systems. In their essay, "Dialogue Games in Multi-Agent Systems," they review the problems and issues arising within the interface of computational linguistics and Argumentation Theory.

Michael A. Gilbert and Chris Reed's paper. "E-motion: Moving Toward the Utilization of Artificial Emotion," addresses a pressing issue in human-machine interactions. Humans, unlike computational systems, invariably have at least some minimal emotional issues or expressions that arise during a dispute. Since computers are famously non-emotional, how can this be incorporated into the mix? It is suggested that by using techniques from discourse analysis, language alteration, linguistic clues, and other, syntactic means, computers can begin to understand when a situation has become emotional.

The final essay in the edition, "Argument Representation for Dependable Computer-Based Systems," is contributed by Corin Gurr, and is an examination of the kinds of arguments that must be addressed in confronting computer safety issues. There are many instances when computer failure can possibly, or even certainly, lead to the loss of human life. Gurr argues that a meta-logical framework incorporating tools from both formal and informal logic can move us to a surer 
and safer system of dependability arguments. It is crucial that the fullest and richest representational models be available to insure that human agents can assess and monitor the control of agent systems.

The papers collected here are at the leading edge of the investigation of Natural Language Processing utilizing argumentation. With the exception of Hitchcock and Gilbert, who writes with Reed, all the essays are written by computer experts who are drawing upon Informal Logic and Argumentation Theory in order to advance the field. The issues dealt with here are only a few of those that must be considered if progress is to be made. Concerns such as the identification of argument forms, the recognition of argumentative moves, fallacies, the role of argument in computer-human interactions, all require considerable further exploration. We recognize that the collection here tends to the technical and may involve concepts somewhat foreign to the reader. Nonetheless, we urge you to examine them and become familiar with the issues and basic notions in the intersection of Artificial Intelligence and Informal Logic. There is much to be done, and the contributions will come from far and wide.

I wish to express my heartfelt thanks to the editors of Informal Logic for the opportunity to bring these papers together. It is important that Informal Logic move forward and expand its limits so as to remain relevant and continue contributing to human knowledge as well as human pedagogy. This is courageous and warrants recognition. I would also like to thank the contributors for bringing together what I hope will be received as an interesting and exciting set of essays.

\section{References}

Perelman, C. and Olbrechts-Tyteca, L. 1969. The new rhetoric: A treatise on argumentation. Notre Dame, IN: University of Notre Dame.

Pollock, J. L. 1999. "Download OSCAR", at http://www.u.arizona.edu/ pollock/oscar.html. 